Z Epileptol 2018 $\cdot 31: 1-2$

https://doi.org/10.1007/s10309-018-0174-1

(c) Springer Medizin Verlag GmbH, ein Teil von Springer Nature 2018

CrossMark

\section{Friedhelm C. Schmitt ${ }^{1}$. Adam Strzelczyk ${ }^{2}$}

' Universitätsklinik für Neurologie, Otto-von-Guericke-Universität, Magdeburg, Deutschland

${ }^{2}$ Epilepsiezentrum Frankfurt Rhein-Main, Universitätsklinikum Frankfurt, Frankfurt, Deutschland

\title{
„Ein Blick zurück und nach vorne“
}

Den Beginn des Jahres wollen wir mit einem Rückblick beginnen. Die ersten beiden Themenhefte diesen Jahres gehen - durch die hervorragende und vorrausschauende Arbeit - noch auf unsere Vorgänger Herrn Prof. B. J. Steinhoff (Kork) und Frau Prof. H. Potschka (München) zurück. Wie auch in den letzten Jahren werden im Jahr 2018 wieder interessante Themen in der Zeitschrift für Epileptologie von Gastherausgebern bearbeitet werden: PD Dr. R. Surges (Aachen) und PD Dr. T. von Oertzen (Linz, Österreich) beschäftigen sich zusammen mit ihren Koautoren mit einigen der wichtigsten „Komorbiditäten“ in der Epileptologie. Prof. J. Wellmer (Bochum) und Prof. S. Knake (Marburg) werden mithilfe ihrer Koautoren eine umfangreiche und aktuelle Zusammenstellung von Artikeln über das so wichtige Thema „Bildgebung" herausgeben. Im Anschluss daran wird sich das dritte Themenheft von Prof. S. Beniczky (Aarhus, Dänemark) und PD Dr. S. Rampp (Erlangen) allgemeinverständlich, u.a. mithilfe von Fallberichten, dem Thema „Quellenlokalisation“ nähern. Die beiden zuletzt genannten Themenschwerpunkte haben eine besondere Rolle, wenn es um die Indikationsstellung für operative Therapiemöglichkeiten geht. Die Bildgebung ist essenziell für die Epilepsiechirurgie, die Quellenlokalisation wird oft als schwierig wahrgenommen, kann jedoch wichtige Zusatzinformationen liefern. Im letzten Themenheft dieses Jahres werden PD Dr. C. Kellinghaus (Osnabrück), Prof. E. Trinka (Salzburg, Österreich) und Prof. A. Strzelczyk (Frankfurt a. M.) mit ihren Koautoren zusammen das Thema "Status epilepticus“ aufbereiten. Da sich hier seit dem letzten Heft (02/2013) $\mathrm{zu}$ diesem Thema einiges Neues getan hat: Die neue Definition der ILAE wurde zwischenzeitlich publiziert [1], es sind neue Kriterien zur Diagnose des nonkonvulsiven Status epilepticus verfügbar, und die Definition eines superrefraktären Verlaufes fand vermehrt Einzug in Studien und klinischen Alltag.

Besonders erfreut uns, dass - wie auch in den letzten Jahren zuvor - allen Heften gemein ist, dass sie von Autoren und/oder Gastherausgebern gestaltet werden, die über die Landesgrenzen Deutschlands reichen. Zudem werden auch wieder einige englischsprachige Artikel erscheinen. Wir hoffen, dass sich dieser Trend weiter fortsetzt.

Auch dachten wir uns, dass es im Rahmen eines Rückblicks interessant sein könnte zu schauen, welche Artikel ein besonderes Interesse in der Leserschaft gewonnen haben. Neben der Anzahl der Zitierungen (eine sicherlich wichtige, aber im deutschsprachigen Raum nicht leicht zu erhebende Angabe) kann hierzu die Anzahl der Downloads (alle Stand 20.12.2017) ein „objektives“ Kriterium sein. Bzgl. der Artikel, die in den letzten 5 Jahren erschienen sind, ist hierbei der Artikel „Therapie von Epilepsien im Kindes- und Jugendalter"von PD Dr. T. Bast (Kork) aus dem Themenheft 3 „State of the Art"von 2013 mit 459 Downloads führend [2]. Auf Platz 2 und respektive Platz 3 sind zum einem der englischsprachige und bislang einzige frei downloadbare Artikel „Experimental models for the study of hormonal changes in epilepsy“ [3] von E. Taubøll, K. Heuser, L. Sveberg und S. Svalheim aus dem Themenheft 4/2015 „Epilepsie und Endokrinologie“ (432 Downloads) und der Artikel „Der Epilepsiehund Traumtänzerei, Tierquälerei oder sinnvoller Einsatz? - Systematische Literaturübersicht" von Herrn R. Wohlfarth (Freiburg) und Kollegen (Heft 2/2013,
396 Downloads; [4]). Schaut man ausschließlich auf das vergangene Jahr, haben folgende 3 Artikel die häufigsten Downloads erfahren: A. Strzelczyk, C. Gaul, F. Rosenow und G. Kurlemann's „Visuelle Auren im Grenzgebiet zwischen Epilepsie und Migräne“ [5], Prof. H.-M. Meinck's „Myoklonus - eine Orientierungshilfe“ ([6]; beide aus dem Themenheft 1/2017 „The Borderland of Epilepsy“; 146 Downloads bzw. 130 Downloads) und T. Polster, R. Schulz, F.G. Woermann, M.K. Bernhard, F.C. Schmitt, L. Büntjen und J. Voges's „Thermoablation bei nodulären Heterotopien. 2 Fallberichte mit Verlauf über mehr als 5 Jahre" (aus dem Themenheft 2/2017 „Minimalinvasive Ablationsverfahren in der Epilepsiechirurgie“; 129 Downloads; [7]).

Zudem gibt es in diesem Jahr noch einige andere Änderungen: Auch aufgrund einiger spontaner Einsendungen wurde eine neue Rubrik unter der Federführung von PD Dr. B. Kaspar (Erlangen) und Dr. G. Krämer (Zürich, Schweiz) mit dem Themenschwerpunkt „Historisches und Denkwürdiges“ gegründet. Ebenfalls neue konstante Rubrikherausgeber sind Prof. S. Knake (Marburg) und PD Dr. G. Ramantani (Zürich, Schweiz), die sich bereit erklärt haben, Beiträge zu interessanten „epilepsiechirurgischen Fällen“ zusammenzustellen. Ab diesem Jahr möchten wir zudem eine neue Rubrik - analog zu den „Images in Clinical Medicine" im New England Journal of Medicine - beginnen. In der Rubrik „Das klinische Bild“ sollen interessante Befunde aus der Bildgebung und Elektroenzephalographie, aber auch andere Fotos oder downloadbare Videosequenzen veröffentlicht werden, die mit maximal 200 Wörtern und 2 Zitaten eine in- 


\section{Editorial}

teressante epileptologische Fragestellung verdeutlichen sollen.

Wir möchten alle Leser auffordern, die alten und die neuen Rubriken durch Zusendung von Artikeln (gerne auch spontan) zu unterstützen. Abschließend möchten wir uns für die so geduldige und professionelle Zusammenarbeit mit dem Springer Medizin Verlag und insbesondere mit Frau Elster bedanken.

Wir sind gespannt auf die Entwicklung der kommenden Jahre.

Ihre

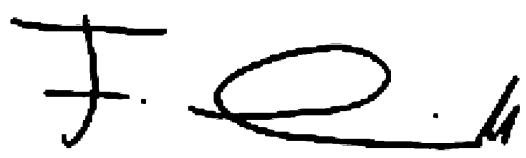

F.C. Schmitt, Magdeburg, und

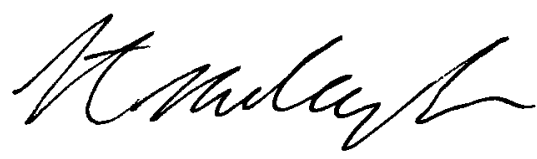

A. Strzelczyk, Frankfurt a. M.

\section{Korrespondenzadresse}

\section{PD Dr. F. C. Schmitt}

Universitätsklinik für Neurologie, Otto-vonGuericke-Universität

Leipziger Str. 44, 39120 Magdeburg,

Deutschland

fc.schmitt@med.ovgu.de

\section{Prof. Dr. A. Strzelczyk, MHBA}

Epilepsiezentrum Frankfurt Rhein-Main,

Universitätsklinikum Frankfurt

Schleusenweg 2-16, 60528 Frankfurt,

Deutschland

strzelczyk@med.uni-frankfurt.de

Interessenkonflikt. F.C. Schmitt und A. Strzelczyk geben an, dass kein Interessenkonflikt besteht.

\section{Literatur}

1. Trinka E, Cock H, Hesdorffer D et al (2015) A definition and classification of status epilepticus-Report of the ILAE Task Force on Classification of Status Epilepticus. Epilepsia 56:1515-1523. https://doi.org/10.1111/epi.13121

2. Bast T (2013) Therapie von Epilepsien im Kindesund Jugendalter. Z Epileptol 26:134-141. https:// doi.org/10.1007/s10309-013-0305-7

3. Taubøll E, Heuser K, Sveberg L et al (2015) Experimental models for the study of hormonal changes in epilepsy. Z Epileptol 28:246-253. https://doi.org/10.1007/s10309-015-0001-x
4. Wohlfarth R, Mutschler B, Bitzer E (2013) Der Epilepsiehund - Traumtänzerei, Tierquälerei oder sinnvoller Einsatz? - Systematische Literaturübersicht. Z Epileptol 26:90-97. https://doi.org/10. 1007/s10309-013-0313-7

5. Strzelczyk A, Gaul C, Rosenow F et al (2017) Visuelle Auren im Grenzgebiet zwischen Epilepsie und Migräne. Z Epileptol 30:21-27. https://doi.org/10 1007/s10309-016-0098-6

6. Meinck HM (2017) Myoklonus - eine Orientierungshilfe. Z Epileptol 30:13-20. https://doi.org/ 10.1007/s10309-016-0087-9

7. Polster T, Schulz R, Woermann FG et al (2017) Thermoablation bei nodulären Heterotopien. 2 Fallberichte mit Verlauf über mehr als 5 Jahre. Z Epileptol 30:122-128. https://doi.org/10.1007/ s10309-017-0107-4 